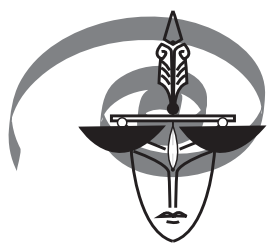

EUROPEAN

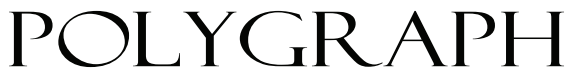

Volume $12 \cdot 2018 \cdot$ Number 2 (44)

DOI: $10.2478 / \mathrm{ep}-2018-0008$

Tuvia Shurany* Liecatcher Polygraph Series Jerusalem, ISRAEL

Nathan J. Gordon* Director Academy for Scientific and Investigative Training, Philadelphia PA, USA

\title{
Idiosyncrasies in Chart Evaluation**
}

\section{Особенности расшифровки полиграмм}

Key words: polygraph chart evaluation, polygraph , changes as reaction criteria, evaluation of chart interpretation

In the 1970's and 1980's, when the authors attended an American Polygraph Association accredited polygraph schools they were taught that the following 33 changes were reaction criteria:

Pneumo: Lower baseline apnea, median apnea, upper baseline apnea, suppression, hyperventilation, respiration slow down, respiration speed up, changes in inhalation/exhalation ratio, ascending escalations (upward staircase), descending escalations (downward staircase), ascending escalation followed by descending escalation (half-moon), descending escalation followed by ascending escalation (reverse half-moon), descend-

*tuvia@liecatcher.com

*nategordon@isope.net

** "Reprinted from "W poszukiwaniu prawdy. Księga jubileuszowa ofiarowana Profesorowi Janowi Widackiemu” ( Search of the Truth - Tome for Jubilee of Professor Jan Widacki) Oficyna AFM, Kraków 2018”. 
ing escalations (downward staircase), ascending escalation followed by descending escalation in hyperventilation (half-moon), descending escalation followed by ascending escalation in hyperventilation (reverse half-moon) baseline arousal, and baseline drop.

EDA: Amplitude of reaction, duration of reaction, complexity of reaction, and a plunging tracing (Devil's finger).

Cardio: Blood pressure/volume increase, blood pressure/volume decrease, combination of increase/decrease, combination of decrease/increase, slow down of heart rate, increase in heart rate, upward shift of the dicrotic notch, downward shift of the dicrotic notch, increase in tracing amplitude, decrease in tracing amplitude, premature ventricular contractions (PVCs, extended diastole, and extended systole.

In other words, any change from the examinees homeostatic norm that was not associated with distortion was considered reaction criteria, and where there were changes in both the Comparison and Relevant Questions the examiner decided the change that held the greatest threat to survival was the greater reaction. For example, if the Comparison Question showed a pattern of minor suppression and the Relevant question showed apnea, no air was a greater threat to survival than less air and was considered to be the stronger reaction.

A strong opponent to this view that any change from the homeostatic norm was reaction criteria was Cleve Backster, who separated changes from homeostatic norm into reaction criteria and relief criteria. Backster maintained that where there is relief, there cannot be reaction. If a question showed a relief reaction, such as hyperventilation in the pneumo, even if there was absolutely no change in the pneumo pattern to which it was compared, Backster would have determined the pattern without any change was $\mathrm{a}+$ or -1 , and labeled this as "Reaction by Deduction".

James Allen Matte, in his extensive 1996 text [1] identifies the following 26 changes as reaction criteria:

Pneumo: Ascending suppressed cycle, sustained suppressed cycle, upper baseline apnea (holding), Lower baseline apnea (blocking), descending suppressed cycles, baseline arousal, baseline drop, baseline arousal, return from a dropped baseline, decrease in breathing rate, change in inhalation/exhalation ratio, sustained hyperventilation, ascending hyperventilation cycles, descending hyperventilation cycles, increased breathing rate.

EDA: Tracing excursion (amplitude), complexity and duration. 
Cardio: Blood pressure arousal to include: sustained blood pressure trend, ascending blood pressure trend, amplitude increase, amplitude decrease, increase in pulse rate, decrease in pulse rate, extended diastole, extra systole (PVCs), dramatic decrease in pulse amplitude, irregularity in pulse, upward change in the positioning of the dicrotic notch, downward change in the positioning of the dicrotic notch.

In 1999, Jimmie Swinford published "Manually Scoring Polygraph Charts Utilizing the Seven-Positioning Numerical Analysis Scale at the Department of Defense Polygraph Institute”. [2] In that article he identifies the following 22 reaction criteria:

Pneumo: Respiration rate decrease, respiration rate increase, respiration inhalation/ exhalation ratio change, respiration amplitude increase, respiration suppression, progressive increase followed by a decrease, progressive increase and return to homeostasis, progressive decrease and return to homeostasis, temporary respiration baseline change, permanent respiration baseline loss, upper baseline apnea or holding of breath, and lower baseline apnea or blocking.

EDA: Amplitude of reaction, complexity of reaction, and duration of reaction.

Cardio: Phasic increase and decrease in baseline, tonic increase in baseline, tonic decrease in baseline, increase in amplitude, decrease in amplitude, increase in rate, decrease in rate, premature ventricular contractions (PVCs).

In, "The Frequency of Appearance of Evaluative Criteria in Field Polygraph Charts" [3], by Norman Ansley and Donald Krapohl they report a study into the frequency of 22 response patterns. Interestingly, in reviewing 616 polygraph charts of 177 cases they found that there was a reduction of reactions in all three components in the $2^{\text {nd }}$ and $3^{\text {rd }}$ charts of non-deceptive examinees, however only in the EDA and cardio of deceptive examinees, with an increase in pneumo reactions in later charts for deceptive examinees. They also found that deceptive examinees produced higher tonic heart rates than non-deceptive examinees. They did not report observations dealing with changes in the overall trend of reactions taking place as charts were administered between their truthful and deceptive populations. The criteria they researched were the criteria Swinford reported in 1999. They reported that the pneumo was credited with $19 \%$ of the observed reactions, the EDA $55 \%$ and the cardio 26\%. They found the frequency of reactions as follows:

EDA amplitude changes $26 \%$

EDA duration $24 \%$

Cardio baseline increase and decrease $15 \%$

EDA complex reactions $6 \%$

Cardio amplitude decreases 5\% 
Pneumo amplitude increases $4 \%$

Pneumo temporary baseline changes $4 \%$

Cardio baseline increases 3\%

Pneumo suppressions 3\%

Pneumo permanent baseline changes 2\%

Pneumo rate decreases 2\%

Pneumo progressive decrease and return 1\%

Pneumo apnea at exhalation 1\%

Pneumo rate increase 1\%

Pneumo progressive increase and return $1 \%$

Pneumo progressive increase/decrease 1\%

I:E ratio changes .5\%

Cardio rate increase .5\%

Cardio rate decrease .5\%

Pneumo apnea at inhalation .5\%

Cardio amplitude increase .5\%

Based on this study, Ansley and Krapohl suggested that the list of evaluative criteria could be shortened for chart interpretation, which appears to be the basis for the "Defensible Dozen" criteria for data analysis.

The "Defensible Dozen" along with the frequency of occurrence found in the previously mentioned study are:

Pneumo: Upper baseline apnea (.5\%), suppression (3\%), progressive decrease (1\%), respiration slow down (2\%), I:E ratio changes $(.5 \%)$, and a temporary increase in baseline $(4 \%)$.

EDA: Amplitude (26\%), complexity (6\%) and duration (24\%).

Cardio: Baseline/blood pressure increase (15\%), response duration (Not Reported), and a decrease in heart rate (.5\%).

A question concerning the results of this study is what was the reasoning for the selection of these finalized 12 criteria? We can see based on the Ansley and Krapohl study that some of the eliminated criteria had the same frequency as the criteria selected. Were the reductions due to simplifying what computerized algorithms could identify? Was it because polygraph examiners are not intelligent enough to analyze any changes from the norm and the threat to survival? Certainly it could not be what is defensible in a court of law, since very few polygraph examinations were or are entered into court 
evidence, and based on the 2000 study mentioned above some of the criteria observed at a .5 frequency were kept while others were eliminated.

How can we define what physiological changes will occur when an individual is afraid? Some people experiencing fear may run, some may faint, and some may urinate. Individual reaction capability is just that, individual. In addition, eliminating reaction criteria for statistical appearance is incorrect. The world population is approximately 7.5 billion people. There are 12 million individuals reported as suffering from Parkinson's disease. Statistically this disease only affects 0.16 percent of the world's population. Therefore, based on statistics there is no need to find a cure or treat this disease!

In the current research identifiable data characteristics there were changes from the homeostatic norm in the twenty-four (24) examinations utilized, which consisted of eighty-five (85) charts, the following observations and findings were made consisting of 670 in the pneumo tracings, 462 in the EDA and 633 in the Cardio. To highlight reactions not included in the "Defensible Dozen" those meeting their criteria are in bold):

\begin{tabular}{|l|c|c|}
\hline PNEUMO REACTIONS: & Occurance & Percentage \\
\hline Decrease or Slow Down in Rhythm & 149 & $22 \%$ \\
\hline Increase or Speed Up in Rhythm & 5 & $.75 \%$ \\
\hline Suppression/Decrease in Amplitude & 106 & $16 \%$ \\
\hline Increase in Amplitude and Volume & 18 & $2.7 \%$ \\
\hline Inhalation-Exhalation Ratio Changes & 16 & $2.4 \%$ \\
\hline Temporary Baseline Arousal & 63 & $9.4 \%$ \\
\hline Temporary Baseline Loss & 16 & $2.4 \%$ \\
\hline Permanent Baseline Arousal & 18 & $2.7 \%$ \\
\hline Permanent Baseline Loss & 51 & $7.6 \%$ \\
\hline Hyperventilation & 3 & $.45 \%$ \\
\hline Apnea & 20 & $3 \%$ \\
\hline Ascending Escalation/Upward Staircase & 70 & $10.5 \%$ \\
\hline Descending Escalation/Downward Staircase & 62 & $9.25 \%$ \\
\hline
\end{tabular}




\begin{tabular}{|l|c|c|}
\hline EDA REACTIONS: & Occurance & Percentage \\
\hline Amplitude/Vertical Increase & 72 & $80.5 \%$ \\
\hline Complex Reaction & 87 & $19 \%$ \\
\hline Increased Durations & 3 & $.65 \%$ \\
\hline Desplome del Trazo del EDA?? & 0 & $0 \%$ \\
\hline
\end{tabular}

\begin{tabular}{|l|c|c|}
\hline CARDIO REACTIONS: & Occurance & Percentage \\
\hline Increase/Decrease in Blood Pressure/Volume & 397 & $62.7 \%$ \\
\hline Increase Only in Blood Pressure/Volume & 69 & $11 \%$ \\
\hline Decrease Only in Blood Pressure/Volume & 6 & $.95 \%$ \\
\hline Increase in Tracing Amplitude & 1 & $.16 \%$ \\
\hline Decrease in Tracing Amplitude & 138 & $22 \%$ \\
\hline Extra Systole Disappearance & 0 & $0 \%$ \\
\hline Extra Systole Appearance & 1 & $.16 \%$ \\
\hline Change in the Position of the Dichroitic Notch & 13 & $2 \%$ \\
\hline
\end{tabular}

The "Defensible Dozen" accounted for 33\% (223 of the 670) of the observable Pneumo reactions, all of the EDA reactions, and 74\% (466 of the 633) of the Cardio reactions in our research. Overall the "Defensible Dozen" identified 65\% (1,151 of the 1,765) of our observed reactions.

Additionally, this research along with other studies [4] previously conducted bring into serious question as to whether the EDA tracing should automatically be given greater weight than the other channels when analyzing the data. Further research should be done regarding whether automatically giving the EDA greater value is beneficial, and whether "bigger is better" is enough to justify a greater score, or cancel stronger reactions in the pneumo and cardio when the EDA is simply visibly bigger. 
Imagine the following spot reaction:

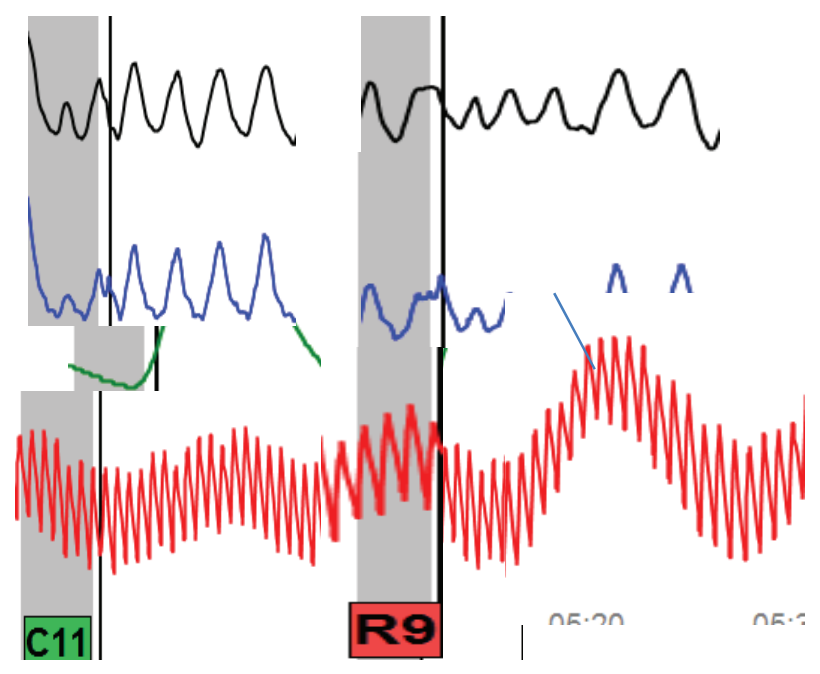

Using a traditional 7 point scale the pneumo tracing would have received a -2 , the EDA 0 and the cardio -2 , totaling a spot score of a -4 . Imagine this was a event specific single issue test with three relevant questions and similar spot scores. Each chart total would have been a -12, and a 3 chart test score totaling -36. Now, let's apply ESS to the same data. The pneumo score $\mathrm{a}-1$, the EDA a +2 and the cardio a -1 , for a total spot score of " 0 " and total exam score of " 0 ." Even a 3 point scale using the "bigger is better" concept, would result in a -1 pneumo, +1 EDA and -1 in the cardio for a spot score of -1 , and an exam score of -9 !

The authors believe that physiological reactions on polygraph charts for the most part are related to fear. Innocent examinees fear that the threat of being non-truthful to the Comparison Questions may cause them to be determined to be deceptive to the test issue, and deceptive examinees fear lying to the Relevant Questions will result in their determination of being deceptive to the test issue. This is Backster's theory and definition of Psychological Set. [5] While the deceptive examinee is lying to both the Comparison and Relevant Questions, the Relevant Questions will create greater reactions due to Backster's theory of Anti-Climax Dampening Concept [6] (the greater threat reduces a person's ability to react to lesser threats). These "fear patterns" are related to the three F's (freeze, fight, flight) which are the principle ways humans react to threat. 


\section{Conclusion}

The "Defensible Dozen" based on our research properly identifies two-thirds of the valid physiological reactions occurring in polygraph examinations. However, one third of the reactions fall outside of its definition based more on idiosyncratic individual reaction patterns. Lower frequency in response appearance does not indicate lack of existence! The "Defensible Dozen" is an excellent starting point for new professionals in the field; however as examiners progress in their development and goal to make the most accurate determinations possible, examiners must be familiar with these idiosyncratic individual reaction patterns and them into their decision making process.

\section{References}

[1] Matte J.A., 1996, Forensic Psychophysiology Using The Polygraph, New York, 373-390.

[2] Swinford J., 1999, Manually Scoring Polygraph Charts Utilizing the Seven-Positioning Numerical Analysis Scale at the Department of Defense Polygraph Institute, "Polygraph", 28, 1, 10-27.

[3] Ansley N., Krapohl D. 2000, The Frequency of Appearance of Evaluative Criteria in Field Polygraph Charts, "Polygraph", 29, 2, 169-176.

[5] Matte J.A, Reuss R. 1992, A Study of the Relative Effectiveness of Physiological Data in Field Polygraph Examinations, "Published in Polygraph", 21, 1; Gordon N.J. et al., 2005, Integrated Zone Comparison Polygraph Technique Accuracy with Scoring Algorithms, "Physiology and Behavior".

[5] Backster C., 1969, Technique Fundamentals of Tri-Zone Polygraph Test, Backster Research Foundation, New York.

[6] Backster C., 1974, Anticlimax Dampening Concept, "Polygraph“, 3, 1, 48-50. 